\title{
Correction to: A Tri-level Programming Framework for Modelling Attacks and Defences in Cyber-Physical Systems
}

Waleed Yamany, Nour Moustafa, and Benjamin Turnbull

\section{Correction to:}

Chapter "A Tri-level Programming Framework for Modelling Attacks and Defences in Cyber-Physical Systems" in: M. Gallagher et al. (Eds.): AI 2020: Advances in Artificial Intelligence, LNAI 12576, https://doi.org/10.1007/978-3-030-64984-5_8

The original version of this chapter was revised. The following corrections have been incorporated:

- Typographical errors throughout the paper have been corrected.

- Table 1 has been corrected.

- Eqs. 2 and 3 have been corrected; Eqs. 17, 18, and 19 have been removed.

- An acknowledgement has been added.

- Ref. 9 has been corrected. 